\title{
Outcomes and survival following lung transplantation in non-cystic fibrosis bronchiectasis
}

\author{
Frederick Jung $\mathbb{D}$, Leonard Riley (1) and Jorge Lascano (1)
}

Dept of Internal Medicine, Division of Pulmonary, Critical Care, and Sleep Medicine, University of Florida College of Medicine, Gainesville, FL, USA.

Corresponding author: Frederick Jung (Frederick.Jung@medicine.ufl.edu)

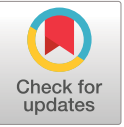

Copyright @The authors 2022

This version is distributed under the terms of the Creative Commons Attribution Non-Commercial Licence 4.0. For commercial reproduction rights and permissions contact permissions@ersnet.org

Received: 27 Oct 2021 Accepted: 21 Dec 2021

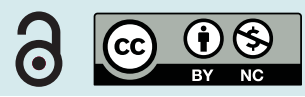

Shareable abstract (@ERSpublications)

A retrospective analysis using UNOS data showed that median survival post-LT for patients with non-CF bronchiectasis is 6.0 years, which is similar to an age- and sex-matched cohort https://bit.ly/3eSv95p

Cite this article as: Jung F, Riley L, Lascano J. Outcomes and survival following lung transplantation in non-cystic fibrosis bronchiectasis. ERJ Open Res 2022; 8: 00607-2021 [DOI: 10.1183/23120541.00607-2021].

\section{Abstract}

Disease-specific outcomes in patients with non-cystic fibrosis bronchiectasis following lung transplantation are not well described.

We performed a retrospective analysis to describe outcomes in these patients. Patients with non-cystic fibrosis bronchiectasis who have undergone lung transplantation in the USA were identified using the Organ Procurement and Transplant Network database. Survival data were analysed for the post-lung allocation score period with Kaplan-Meier curves, and a log-rank test was conducted to compare survival data among an age-, sex- and activation date-matched non-cystic fibrosis bronchiectasis cohort.

721 patients with non-cystic fibrosis bronchiectasis were listed for lung transplantation between March 1992 and September 2019. 407 patients received lung transplantation with a median age at listing of 47 years. The Kaplan-Meier survival analysis for lung transplantation recipient non-cystic fibrosis bronchiectasis patients during the post-lung allocation score period at 1,5 and 10 years was $87 \%$, 53\% and $16 \%$, respectively. The median survival time post-lung transplantation is 6.0 years (interquartile range: $2.3-$ 11.9 years), which is similar to an age- and sex-matched cohort $(\mathrm{p}=0.86)$.

This retrospective analysis demonstrates that median survival after lung transplantation in non-cystic fibrosis bronchiectasis was similar to other lung transplantation recipients over the study period. We suggest that the development of specific criteria for lung transplantation in non-cystic fibrosis bronchiectasis may improve patient selection and benefit a larger group of patients with this therapy.

\section{Introduction}

Bronchiectasis is a disease process in which damage occurs to the bronchial wall leading to an increase in airway diameter [1]. It has many causes, including genetic diseases such as cystic fibrosis (CF) and $\alpha-1$ antitrypsin deficiency, immunodeficiencies and as a sequela of severe pneumonia, among others [2]. Non-CF bronchiectasis (NCFB) differs from CF lung disease in several ways. While CF lung disease is a disease that tends to affect Caucasians from birth, NCFB tends to be recognised in patients in the sixth decade of life [3]. Both CF and NCFB commonly have comorbid conditions. CF is a multiorgan system disease, causing comorbid pancreas, liver and sinus disease, among others [4]. However, the comorbid conditions in NCFB vary and may not be related to the underlying aetiology of bronchiectasis. These diseases may also show different rates of lung function decline. While there are limited head-to-head studies, registry data have shown that per cent predicted for forced expiratory volume in $1 \mathrm{~s}\left(\mathrm{FEV}_{1}\right)$ declines at a rate of $1.45-1.92 \%$ predicted per year in CF [5], whereas in NCFB per cent predicted $\mathrm{FEV}_{1}$ declines at about $0.98 \%$ predicted per year [6].

People with bronchiectasis may be asymptomatic or have shortness of breath, persistent sputum production, recurrent infections, decreased exercise capacity and an obstructive pattern on spirometry [7, 8]. As the disease progresses and lung function declines, patients usually experience worsening quality of life and are 
at increased risk of death [8]. While there are treatments for bronchiectasis, including airway clearance therapies, treatment of infections and treatment of the underlying aetiology [7], there is no known cure.

Lung transplantation is a therapy for end-stage NCFB. It makes up a small proportion of lung transplants performed, as only 4.5\% of all bilateral lung transplants were performed for NCFB between January 1995 and June 2008 [1]. Several centres have reported their own experience with lung transplantation in the NCFB population, but these outcomes are not well described on a national scale. TitMAN et al. [9] described lung transplantation outcomes in a national cohort of patients with bronchiectasis in the UK, but a similar cohort in the USA has not been published. This study sought to describe disease-specific outcomes in patients with NCFB following lung transplantation in the USA.

\section{Materials and methods}

This was a retrospective study using database-derived data that sought to describe mortality outcomes for lung transplantation for NCFB. This study was approved by the University of Florida Institutional Review Board (IRB202002417). Data were extracted from the Organ Procurement and Transplant Network (OPTN) database maintained by the United Network for Organ Sharing (UNOS) regarding all lung transplant candidates in the USA between March 1992 and September 2019. Patients listed within 4 years of the study end date were not included in the wait list or survival analysis to prevent right censoring. Survival data were analysed for the post-lung allocation score (LAS) period with Kaplan-Meier curves, and a log-rank test was conducted to compare survival data among an age-, sex- and activation date-matched NCFB cohort. To create an age-, sex- and activation date-matched cohort, a case-matched method was used. The dataset was divided according to sex and then sorted by descending age and secondly by descending activation date. The subject with the closest activation date to the NCFB subject was selected as the matched case. This method was used over a multivariable model to adjust for the activation date more accurately, since disease treatment and survival are linked to time.

A Fisher's exact test was used to compare the post-lung transplantation rates of airway dehiscence, dialysis and stroke between the NCFB and all lung transplantation recipients, as well as the NCFB cohort and the matched cohort. Interquartile range (IQR) was defined as the 25th to 75th percentile of the entire range of the cohort. Patients who received a lobar or multiorgan transplant were not included in the analysis. The significance level for all tests was $\alpha<0.05$. Analyses were performed with JMP, version 15.0 (SAS Institute, Cary, NC, USA).

\section{Results}

A total of 721 patients with NCFB were listed for a lung transplantation, and 407 patients received a lung transplantation. The baseline demographic, clinical and physiological characteristics of listed and transplanted recipients are summarised in table 1 . Over the same time period, the total number of patients listed for lung transplantation for all indications was 60872, and 39864 (65.2\%) were transplanted. During the LAS period, 34670 patients were listed, and 25725 (74.2\%) were transplanted. At the time of data analysis, 263 patients who were transplanted for NCFB had died (65\%), 114 patients were alive (28\%), 16 patients were lost to follow-up (4\%) and 14 patients were retransplanted (3\%) (table 2). The median waiting-list time for subjects who were transplanted was 254 days (IQR 83-568 days), and since the institution of the LAS in May of 2005, the median waiting-list time has been 119 days (IQR 36-301 days).

TABLE 1 Baseline characteristics of NCFB listed and transplant recipients

\begin{tabular}{|c|c|c|}
\hline Variable & Listed patients & Transplanted patients \\
\hline Age years & $47(37-55)$ & $47(37-56)$ \\
\hline Sex, female & $431(60)$ & $221(54)$ \\
\hline $\mathrm{BMI} \mathrm{kg} \cdot \mathrm{m}^{-2}$ & $21.8(18.9-25.2)$ & $21.8(18.8-25.1)$ \\
\hline $\mathrm{FEV}_{1} \%$ predicted & $24(19-31)$ & $24(19-31)$ \\
\hline FVC \% predicted & $41(33-51)$ & $41(33-51)$ \\
\hline Lung allocation score & $34.9(32.8-37.6)$ & $36.9(34.3-43.8)$ \\
\hline Waiting-list time days & $337(110-808)$ & $254(83-568)$ \\
\hline Waiting-list time post-LAS days $(n=202)$ & $170(54-450)$ & $119(36-301)$ \\
\hline
\end{tabular}


TABLE 2 Overall number depicting the type of transplant and the outcomes following lung transplantation in the cohort of 407 non-cystic fibrosis bronchiectasis patients

\begin{tabular}{lc} 
Outcome & $\mathbf{n}(\%)$ \\
\hline Alive & $114(28)$ \\
Dead & $263(65)$ \\
Missing data & $16(4)$ \\
Retransplanted & $14(3)$ \\
\hline
\end{tabular}

Single lung transplantation was performed in 11 patients (3\%), whereas double lung transplantation was performed in 396 patients (97\%). The median length of hospital stay after transplant was 18 days (IQR 1232 days). Prior to discharge 20 patients $(5 \%)$ required dialysis $(p=0.59), 10$ patients $(3 \%)$ had a stroke $(\mathrm{p}=0.48)$ and nine patients $(2 \%)$ developed airway dehiscence $(\mathrm{p}=0.13)$, which are similar to rates in patients who were transplanted for other indications. These findings were similar when the NCFB subjects were compared to the matched cohort regarding dialysis $(p=0.19)$, stroke $(p=0.47)$ and airway dehiscence $(\mathrm{p}=0.55)$.

Details regarding the aetiology of death for the 263 deceased NCFB recipients were reviewed. The most common causes of mortality were graft failure (64 out of 263, 24\%), infection (59 out of 263, 23\%) and respiratory failure (49 out of 282, 19\%) (table 3). Infection accounted for 19.1\% (3876 out of 20310) of the reported deaths for all subjects transplanted without NCFB, which was similar to the NCFB cohort (OR 0.81, CI 0.61-1.09, $\mathrm{p}=0.18$ ).

The median survival time post-lung transplantation in the LAS period was 6.0 years (IQR 2.3-11.9 years). The Kaplan-Meier survival for all 114 patients transplanted for NCFB in the post-LAS period in our study was $87 \%, 53 \%$ and $16 \%$, at $1-$, 5- and 10 years, respectively (figure 1 ). The median survival in the post-LAS period for the age-, sex- and activation date-matched cohort was 5.5 years (IQR 2.3-13.2 years) (table 4). A Kaplan-Meier curve demonstrated similar survival for patients transplanted for NCFB compared to the matched cohort $(\mathrm{p}=0.86)$ (figure 1$)$.

\section{Discussion}

Lung transplantation is an accepted therapy for end-stage NCFB, but outcomes are not well-described in the USA due to low volume and single-centre reporting. This study shows that survival of patients undergoing lung transplantation for NCFB in the USA from institution of the LAS to September 2019 is similar to the survival of an age-, sex- and activation date-matched cohort of patients undergoing lung transplantation for other indications. This study also describes the most common causes of death in patients undergoing lung transplantation for NCFB.

Since this is a retrospective study of registry data, some of the specifics regarding lung transplantation are unavailable. For example, it has been shown that the microbiology of the airway before transplant can affect outcomes after lung transplantation. MATTNER et al. [10] demonstrated in a German cohort that patients colonised with gram-negative rods prior to transplant had a higher risk of post-operative pneumonia. In the cohort from BIRCH et al. [11], the only patients who developed Pseudomonas aeruginosa colonisation at one year were those who were colonised with $P$. aeruginosa prior to their transplant. In the CF population, it has also been demonstrated in a small cohort that $P$. aeruginosa isolates after transplant match those isolated pre-transplant, suggesting a within-host source [12]. A study by Willner et al. [13] demonstrated that in patients transplanted for CF, recolonisation of the donor lungs with $P$. aeruginosa is not associated with bronchiolitis obliterans syndrome (BOS). However, de novo

TABLE 3 Summary of survival for lung transplant recipients in the post-LAS period for NCFB compared to an age- and sex-matched cohort

\begin{tabular}{lcccc} 
Group & Median (IQR) years & 1-year survival \% & 5-year survival \% & 10-year survival \% \\
\hline NCFB & $6.0(2.3-11.9)$ & 87 & 53 & 16 \\
Matched cohort & $5.5(2.3-13.2)$ & 84 & 55 & 28 \\
\hline \multicolumn{4}{l}{ LAS: lung allocation score; NCFB: non-cystic fibrosis bronchiectasis; IQR: interquartile range. } \\
\hline
\end{tabular}




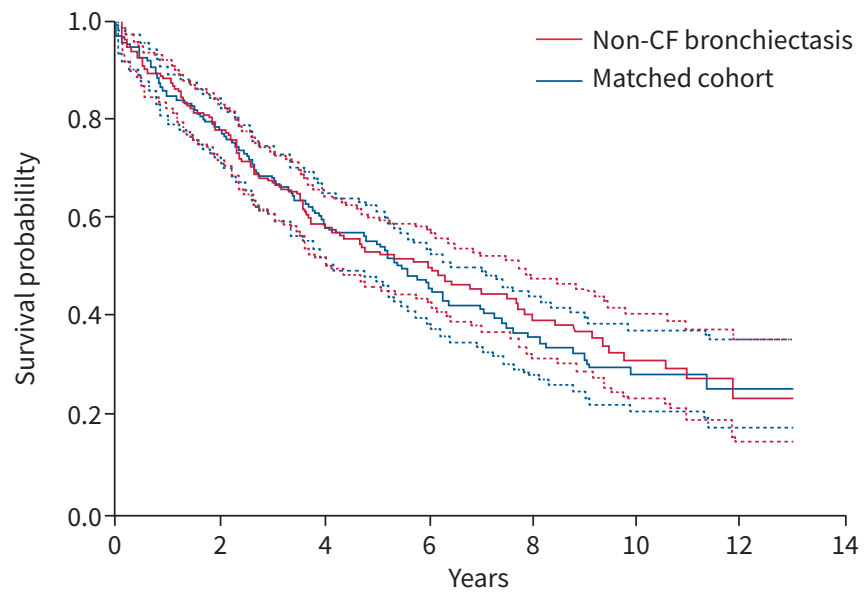

FIGURE 1 Kaplan-Meier survival curve of patients transplanted for non-cystic fibrosis bronchiectasis (solid red line) compared to lung transplant recipients for all other diseases combined (solid blue line) with $95 \%$ confidence intervals (dashed lines).

$P$. aeruginosa colonisation of the airway is associated with development of BOS [14]. All of the above demonstrates that the microbiome that develops in the transplanted lung can influence outcomes after transplant. Unfortunately, these pre-transplant microbiological data were not available for review, so we are unable to assess how differences in airway microbiology may have affected mortality.

Several centres have reported their own experience with lung transplantation in the NCFB population, but these studies have lower patient volumes, ranging in patient numbers from 20 to 54 patients. RusANOv et al. [15] published their experience from a single institution over 16 years in Israel comparing lung transplantation for NCFB to lung transplantation for CF lung disease. Their NCFB group of 33 patients had a median survival of 7.1 years post lung transplantation, compared to 8.4 years in the CF lung disease group, a difference that did not reach statistical significance. They also performed a Cox proportional hazard analysis to examine risk factors for mortality, finding that going on cardiopulmonary bypass during surgery was a risk factor for mortality in patients with NCFB. In general, they found that the outcomes for lung transplantation for NCFB and CF lung disease were similar.

BiRCH et al. [11] also published their experience performing lung transplantation for NCFB. They found that the median survival post-lung transplantation for their cohort of 42 patients was 9.3 years, which was comparable to survival in lung transplantation for other indications at their centre. Several abstracts have been presented recently, which also report single-centre experience with lung transplantation for NCFB. KenNEDY et al. [16] found that the median survival at their institution in Australia was 38\% at 5 years and noted that this was much worse than their survival for patients transplanted for CF lung disease.

TABLE 4 Aetiology of death for non-cystic fibrosis bronchiectasis lung transplantation recipients

\begin{tabular}{lc} 
Aetiology of death & $\mathbf{n}(\%)$ \\
\hline Graft failure & $64(24)$ \\
Infection & $59(23)$ \\
\hline Respiratory failure & $49(19)$ \\
Unknown & $39(15)$ \\
Multiple organ failure & $14(5)$ \\
Cardiovascular & $12(5)$ \\
Malignancy & $11(4)$ \\
Cerebrovascular & $6(2)$ \\
Haemorrhage & $4(2)$ \\
Renal failure & $4(2)$ \\
Suicide & $1(0.4)$ \\
\hline
\end{tabular}


DANTAS et al. [17] from Portugal found that the survival at their institution at 5 years was $79 \%$ and noted that this was better than the survival for other indications. It is unclear what accounts for this dramatic difference in outcomes. The largest study available is from TrTMAn et al. [9], which evaluated outcomes of lung transplantation for all diseases in the UK from 1995 to 2006. Their bronchiectasis cohort was made up of 54 patients, and their median survival was 8.2 years.

Our data agree with what others have found. Survival for patients transplanted for NCFB tends to be comparable to survival for all other indications for lung transplantation. These patients are transplanted in low numbers, yet our study demonstrates that their survival is similar to other patients who undergo lung transplantation.

In many ways, experience from lung transplantation in the CF population is applied to the NCFB patient population. For example, listing criteria and expected outcomes are often extrapolated to the NCFB population from patients with CF [1]. The International Society for Heart and Lung Transplantation guidelines for lung transplant recipient selection either do not give guidance regarding recipients with NCFB [18] or NCFB is combined with CF lung disease, with the admission that data for NCFB are limited [19, 20].

In addition, we found an age discrepancy for transplanting the NCFB population. The average age of diagnosis for these patients is 57 years old [3], yet our study found that the average age of lung transplantation listing was actually 10 years younger, at 47 years old. This age discrepancy suggests a few important points. First, it suggests a selection bias for referred and transplanted subjects. It is possible that the lack of listing criteria specific to bronchiectasis leads to fewer patients being referred to transplant and a more stringent screening process. A more stringent screening process for transplantation may produce transplant candidates that are younger and have fewer comorbidities. Second, registry data show that patients with CF tend to have lung transplantation at a younger age. SETHI et al. [21] found in the UNOS registry that the average age of patients undergoing lung transplantation for CF was 31 years, much lower than the average of 47 years for the NCFB patient group. It is possible that younger patients with NCFB are more likely to meet criteria for lung transplantation listing developed for $\mathrm{CF}$ and thus are referred more often. Criteria that are more specific to NCFB may allow older patients to undergo transplantation.

There is evidence that the application of CF criteria for transplant is poorly applied to the NCFB population. For example, HAYEs et al. [22] demonstrated that patients with advanced NCFB had significantly lower waitlist mortality compared to patients with CF lung disease. Given the differences in demographics and associated comorbidities, such as liver and pancreatic disease in the CF population, these patient groups may be too dissimilar to allow the same criteria to be used for consideration of lung transplant. It may be more appropriate to develop a separate set of criteria for listing patients with NCFB. New bronchiectasis severity scores, such as the Bronchiectasis Severity Index or the FACED [23, 24], may assist in the development of listing criteria for bronchiectasis specifically.

This study has several strengths. First, it is the largest study to evaluate outcomes of lung transplantation in NCFB. There have been several studies reporting single-centre experience in this area, but the patient numbers are small. This study presents survival data on a patient cohort more than twice the size of the largest studies published elsewhere. Second, the data came from multiple centres, which avoid the bias of a single institution's practices and outcomes.

This study does have limitations. It is retrospective in nature. Given the extensive screening process that is used in lung transplantation, the data may be subject to a selection bias. As discussed above, the lack of established guidelines for when to perform lung transplantation for NCFB may make physicians shy away from selecting these patients for lung transplantation. Comparatively, this may mean that younger and otherwise healthier patients undergo lung transplantation for NCFB compared to the patients who undergo lung transplantation for other indications. We sought to avoid this selection bias by comparing survival against age-, sex- and activation date-matched controls.

Another weakness of this study is that it covers a long time period, during which many advances have been made in immunosuppression, surgical techniques and lung allocation. The outcomes presented here are an average over that time and may not reflect the expected outcomes of a patient undergoing lung transplantation for NCFB today. The survival data presented are from the LAS period and are more recent. These survival data are probably more reflective of the outcomes that can be expected today for lung transplantation for NFCB. 
Conclusion

Lung transplantation is a viable option for NCFB. Our study found that in the USA from March 1992 to September 2019, lung transplants for NCFB had similar complications compared to all other indications. Our study also found that in the LAS period, survival for lung transplantation for NCFB was similar to age- and sex-matched controls who were transplanted for another indication. We propose that the development of specific transplant criteria for NCFB may improve patient selection and benefit a larger group of patients with this condition.

Provenance: Submitted article, peer reviewed.

Conflict of interest: None declared.

Support statement: This work was supported in part by Health Resources and Services Administration contract 234-2005-370011C. The content is the responsibility of the authors alone and does not necessarily reflect the views or policies of the Dept of Health and Human Services, nor does mention of trade names, commercial products or organisations imply endorsement by the US Government. Based on OPTN data as of 30 June 2019.

\section{References}

1 Hayes D Jr, Meyer KC. Lung transplantation for advanced bronchiectasis. Semin Respir Crit Care Med 2010; 31: 123-138.

2 O'Donnell AE. Bronchiectasis. Chest 2008; 134: 815-823.

3 Aksamit TR, O'Donnell AE, Barker A, et al. Adult patients with bronchiectasis: a first look at the US Bronchiectasis Research Registry. Chest 2017; 151: 982-992.

$4 \quad$ Elborn JS. Cystic fibrosis. Lancet 2016; 388: 2519-2531.

5 Konstan MW, Wagener JS, Vandevanter DR, et al. Risk factors for rate of decline in FEV in adults with cystic fibrosis. J Cyst Fibros 2012; 11: 405-411.

6 Martinez-García MA, Oscullo G, Posadas T, et al. Pseudomonas aeruginosa and lung function decline in patients with bronchiectasis. Clin Microbiol Infect 2021; 27: 428-434.

7 O'Neill K, O'Donnell AE, Bradley JM. Airway clearance, mucoactive therapies and pulmonary rehabilitation in bronchiectasis. Respirology 2019; 24: 227-237.

8 Loebinger MR, Wells AU, Hansell DM, et al. Mortality in bronchiectasis: a long-term study assessing the factors influencing survival. Eur Respir J 2009; 34: 843-849.

9 Titman A, Rogers CA, Bonser RS, et al. Disease-specific survival benefit of lung transplantation in adults: a national cohort study. Am J Transplant 2009; 9: 1640-1649.

10 Mattner F, Fischer S, Weissbrodt $\mathrm{H}$, et al. Post-operative nosocomial infections after lung and heart transplantation. J Heart Lung Transplant 2007; 26: 241-249.

11 Birch J, Sunny SS, Hester KLM, et al. Outcomes of lung transplantation in adults with bronchiectasis. BMC Pulm Med 2018; 18: 82.

12 Syed SA, Whelan FJ, Waddell B, et al. Reemergence of lower-airway microbiota in lung transplant patients with cystic fibrosis. Ann Am Thorac Soc 2016; 13: 2132-2142.

13 Willner DL, Hugenholtz P, Yerkovich ST, et al. Reestablishment of recipient-associated microbiota in the lung allograft is linked to reduced risk of bronchiolitis obliterans syndrome. Am J Respir Crit Care Med 2013; 187: 640-647.

14 Botha P, Archer L, Anderson RL, et al. Pseudomonas aeruginosa colonization of the allograft after lung transplantation and the risk of bronchiolitis obliterans syndrome. Transplantation 2008; 85: 771-774.

15 Rusanov V, Fridman V, Wille K, et al. Lung transplantation for cystic fibrosis and non-cystic fibrosis bronchiectasis: a single-center experience. Transplant Proc 2019; 51: 2029-2034.

16 Kennedy JL, Ellender C, Steinfort K, et al. Poor outcomes for bronchiectasis patients post lung transplantation. Am J Respir Crit Care Med 2016; 193: A4673.

17 Dantas CDF, Santos AS, Silva D, et al. Lung transplant outcomes in bronchiectasis: a single center experience. Eur Respir J 2019; 54: Suppl. 63, PA1109.

18 Weill D, Benden C, Corris PA, et al. A consensus document for the selection of lung transplant candidates: 2014 - an update from the Pulmonary Transplantation Council of the International Society for Heart and Lung Transplantation. J Heart Lung Transplant 2015; 34: 1-15.

19 Orens JB, Estenne M, Arcasoy S, et al. International guidelines for the selection of lung transplant candidates: 2006 update: a consensus report from the Pulmonary Scientific Council of the International Society for Heart and Lung Transplantation. J Heart Lung Transplant 2006; 25: 745-755.

20 Maurer JR, Frost AE, Estenne M, et al. International guidelines for the selection of lung transplant candidates. The International Society for Heart and Lung Transplantation, the American Thoracic Society, the American Society of Transplant Physicians, the European Respiratory Society. Transplantation 1998; 66: 951-956. 
21 Sethi J, Bugajski A, Patel KN, et al. Recipient age impacts long-term survival in adult subjects with cystic fibrosis after lung transplantation. Ann Am Thorac Soc 2021; 18: 44-50.

22 Hayes D Jr, Kopp BT, Tobias JD, et al. Survival in patients with advanced non-cystic fibrosis bronchiectasis versus cystic fibrosis on the waitlist for lung transplantation. Lung 2015; 193: 933-938.

23 Chalmers JD, Goeminne P, Aliberti S, et al. The bronchiectasis severity index. An international derivation and validation study. Am J Respir Crit Care Med 2014; 189: 576-585.

24 Martínez-García M, de Gracia J, Vendrell Relat M, et al. Multidimensional approach to non-cystic fibrosis bronchiectasis: the FACED score. Eur Respir J 2014; 43: 1357-1367. 